\title{
The configurational energy gap between amorphous and crystalline silicon
}

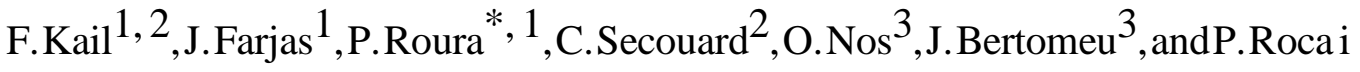 \\ Cabarrocas $^{4}$ \\ 1 GRMT, Department of Physics, University of Girona, Montilivi Campus, 17071 \\ Girona, Catalonia, Spain \\ 2 Univ. Barcelona, Dept. Fisica Aplicada \& Optica, 08028 Barcelona, Spain \\ 3 CEA Grenoble, LTS, 17 rue des Martyrs, 38054 Grenoble cedex, France \\ 4 LPICM, Ecole Polytechnique, 91128 Palaiseau, France
}

* Corresponding author: e-mail pere.roura@udg.es, Phone: +34 97218383, Fax: +34
972418098

Keywords: silicon, crystallization, enthalpy, coordination defects, configurational energy, differential scanning calorimetry

The crystallization enthalpy of pure amorphous silicon (a-Si) and hydrogenated a-Si was measured by differential scanning calorimetry (DSC) for a large set of materials deposited from the vapour phase by different techniques. Although the values cover a wide range $(200-480 \mathrm{~J} / \mathrm{g})$, the minimum value is common to all the deposition techniques used and close to the predicted minimum strain energy of relaxed aSi $(240 \pm 25 \mathrm{~J} / \mathrm{g})$. This result gives a reliable value for the configurational energy gap between a-Si and crystalline silicon. An excess of enthalpy above this minimum value can be ascribed to coordination defects.

In contrast to glasses, whose lower energy states can be accessed by either cooling the liquid at lower rates or by thermal annealing [1], the energy of amorphous tetrahedral semiconductors must be lowered by thermal annealing [2]. Among them, amorphous silicon (a-Si) has been extensively studied due to its technological relevance and because it is usually taken as a model material for covalent amorphous networks.

We have recently shown [3] that after thermal annealing, the rms deviation from the tetrahedral angle, $\Delta \theta$, is approximately $9^{\circ}$ for a broad range of pure and hydrogenated a-Si materials. This "relaxed state" of pure a-Si (no H-atoms remain after annealing) gives support to the theoretical prediction [4] that it is not possible to build stable amorphous models below minimum bond-angle dispersion. In other words, there is a 'configurational gap' between a-Si and c-Si that ensures higher entropy in the amorphous state. This discontinuity makes it impossible for the material to evolve from one state to the other by continuously varying its short-range order.

The bond-angle dispersion entails a built-in strain energy that is released during crystallization [5] and currently detected by differential scanning calorimetry (DSC) as an exothermic peak [6]. By measuring the crystallization enthalpy, $\Delta \mathrm{H}_{\text {cryst }}$, we can, thus, determine the value of the 'configurational energy gap' between a-Si and c-Si. To date, the only systematic measurements of $\Delta \mathrm{H}_{\text {cryst }}$ have been taken from a-Si obtained by ion implantation of c-Si [6] (measurements on hydrogenated thin films being very scarce [7]). Since the experimental values felt within a narrow range (see the right side of Fig. 1), it is generally accepted that they correspond to the minimum energy of a-Si. 
However, since this method of synthesis is very far from equilibrium, there is good reason to suspect that, even after thermal annealing, the material has not reached its lowest energy level. In fact, we suggested [8] that one half of $\Delta \mathrm{H}_{\mathrm{cryst}}$ of this material was due to high concentration of coordination defects and, consequently, predicted that the crystallization enthalpy could be reduced by this factor in defect-free materials.

Recent studies of organic glasses [9] have shown that, thanks to a higher molecular mobility at the film free surface [10], deposition from the vapour phase is a way to achieve low-energy amorphous states. This behaviour could also apply to the deposition of a-Si films from the vapour phase, notably by chemical deposition techniques. So, the aim of this Letter is to reveal, through the careful quantification of $\Delta \mathrm{H}_{\text {cryst }}$ in a large and diverse series of vapour-deposited a-Si materials, the existence of a 'configurational energy gap' between a-Si and c-Si and to quantify its value. In addition, the measured enthalpy values will be used to shed light on the structure of relaxed a-Si.

Approximately twenty a-Si and a-Si:H samples were obtained by various deposition techniques: a) two pure a-Si films on glasss substrates by electron beam evaporation (EBE); b) various a-Si:H films by plasma-enhanced chemical vapour deposition (PECVD) that were collected from the reactor walls in the form of flakes or deposited on glass substrates; c) a-Si:H nanoparticles (np-Si:H) that grew in suspension in the plasma of the PECVD reactor; d) PECVD films and flakes that grew through the simultaneous deposition of nanoparticles and silane radicals, leading to a grade of nanostructured a-Si:H known as polymorphous silicon (pm-Si:H) and, finally, e) four a$\mathrm{Si}: \mathrm{H}$ films by hot-wire CVD (HWCVD) (further details on the samples can be found elsewhere [3]).

The heat of crystallization, $Q_{\text {cryst, }}$ was measured by a DSC of Mettler Toledo (DSC 822). A typical DSC curve is shown in Fig. 2a. At the usual scan rates (0.5-2.5 $\mathrm{K} / \mathrm{min}$ ), 1 to $3 \mathrm{mg}$ were enough to obtain $\mathrm{Q}_{\text {cryst }}$ values with reproducibility better than

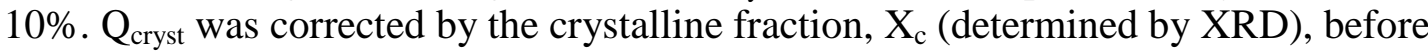
and after the crystallization peak to obtain the crystallization enthalpy, $\Delta \mathrm{H}_{\text {cryst }}$ (Figs. 2a and b). The XRD measurements were taken by using a Smart Apex diffractometer of Bruker AXS. For most samples, $X_{c}=0$ and 1 before and after the crystallization peak, respectively.

The values of $\Delta \mathrm{H}_{\text {cryst }}$ measured for all samples have been collected in Fig. 1. They extend over a wide range: from $195 \mathrm{Jg}^{-1}$ for nanoparticles to $480 \mathrm{~J} / \mathrm{g}$ for $\mathrm{pm}-\mathrm{Si}: \mathrm{H}$ flakes. This picture has been completed by adding the values reported for ion beam amorphized a-Si films (400-450 J/g [6]). Notice that our results modify substantially the previous knowledge about the energy of relaxed a-Si in two main aspects: a) it expands over a much larger range and $b$ ) its minimum value is much lower.

Although, crystallization enthalpy is expected to vary from sample to sample (the amorphous state is not unique but, on the contrary, multiple microscopic configurations are compatible with the mean structure determined by diffraction techniques [11]) we find the experimental variability too large for the following reason. Most of these samples have been characterized by Raman spectroscopy after heating them to $600{ }^{\circ} \mathrm{C}$, resulting in a similar value of $\Delta \theta=9.0^{\circ}$ to $9.7^{\circ}$ depending on the sample [3]. In other words, they are near the (dehydrogenated) 'relaxed state'.

We therefore wished to determine to what extent different values of $\Delta \mathrm{H}_{\text {cryst }}$ can be due to the experimental range of $\Delta \theta$ and if there are hidden structural features that contribute to the variability of $\Delta \mathrm{H}_{\text {cryst }}$. First, first-principle calculations consider the contribution of coordination defects to the energy of a-Si [12]. Second, a 
'paracrystalline model' of a-Si where the paracrystals are topologically crystalline but with highly distorted bond angles has been recently proposed [13].

In fact, the energy of a-Si can be described phenomenologically by the contribution of both the bond-strain energy and that due to coordination defects [5]:

$$
\Delta \mathrm{H}_{\text {cryst }}=\mathrm{U}_{\text {strain }}+\mathrm{U}_{\text {defects }}=\mathrm{A}(\Delta \theta)^{2}+\mathrm{U}_{\text {defects }}
$$

Minor contributions due to bond stretching [8] and to residual Si-H groups can be neglected [3].

Results from our previous studies allow us to predict the value of $\mathrm{U}_{\text {strain }}$ of a-Si just before crystallization. First, we deduced that $A=3.0 \pm 0.3 \mathrm{~J} /\left(\mathrm{g} / \mathrm{deg}^{2}\right)$ [8]. On the other hand, since $\Delta \theta$ falls in the $9.0^{\circ}-9.7^{\circ}$ range after heating to $600{ }^{\circ} \mathrm{C}$ [3], the application of Eq. (1) leads to an expected range of $U_{\text {strain }}=215-310 \mathrm{~J} / \mathrm{g}$ (shaded area in Fig. 1). Notice that 6 samples (and all ion-amorphized layers) have $\Delta H_{\text {cryst }}$ values that are much higher. These deviations are naturally explained as being due to the contribution of defects. Since the formation energy of a dangling bond is approximately $1 \mathrm{eV}$ [14], we obtain a nominal density of dangling bonds of $3 \%$ for every $100 \mathrm{~J} / \mathrm{g}$ in excess of the minimum crystallization enthalpy. Although it is not possible to deduce a reliable absolute defect concentration from our results (since over-coordinated defects, whose formation energy remains unknown, may also exist [12]), they nevertheless constitute the first experimental evidence by calorimetric measurement of high densities of coordination defects in relaxed a-Si. This density is much higher than the experimental density of paramagnetic defects $[2,15]$, what highlights the difficulty to characterize the structure of a-Si.

The variation of $\Delta \mathrm{H}_{\text {cryst }}$ cannot be taken as direct proof of different amounts of paracrystallites because paracrystals are highly distorted and, consequently, their strain energy is similar to that of purely amorphous regions [16].

Anyway, the most important result is that $\mathrm{U}_{\text {strain }}$ is predicted to be $240 \pm 25 \mathrm{~J} / \mathrm{g}$ for the minimum value of $\Delta \theta\left(9^{\circ}\right)$ and, within the error bars, this energy agrees with the lowest values of $\Delta \mathrm{H}_{\text {cryst }}$ measured for any kind of a-Si(:H) material. This value can be interpreted as the energy of relaxed a-Si and free from defects in complete agreement with our former prediction [8]. In other words, we conclude that the energy gap between a-Si and c-Si is around $240 \mathrm{~J} / \mathrm{g}$.

Finally, we wish to discuss one additional result and its significance with respect to the density of defects. The broken lines in Fig. 1 correspond to the values of $\Delta \mathrm{H}_{\text {cryst }}$ measured two and five years after deposition. They reveal an intrinsic instability in those samples with a higher density of defects and show that amorphous solids deposited from the vapour phase suffer from ageing like polymer glasses do [17].

In conclusion, the analysis by DSC of a large set of a-Si(:H) samples grown by different deposition techniques has provided strong evidence of the existence of a configurational energy gap between a-Si and c-Si. According to our results, the minimum energy of a-Si is approximately $240 \mathrm{~J} / \mathrm{g}$. No microscopic configuration of a-Si would be allowed below this value. This value, together with recent heat capacity measurements [18], indicates that, from the thermodynamical point of view, a-Si is much closer to c-Si than it was previously thought. This single value could be used to test the microscopic models of a-Si. Any of the calculations reported so far $[12,16,19]$ give energy values at least three times higher than those found in our experiments. In addition, we have shown that, despite the short range of $\Delta \theta$ values measured before crystallization, the relaxed state that can be effectively achieved by thermal annealing is not unique but depends on deposition conditions. This means that, in contrast with the 
current assumption [20], full relaxation of the bond-angle strain does not ensure that the configuration of minimum energy has been reached. A considerable excess of crystallization enthalpy is probably due to coordination defects at concentrations of several per cent. This is particularly true for the films obtained by ion-beam amorphization whose enthalpy is twice the minimum value. By contrast, most of the materials deposited from vapour phases have crystallization enthalpies close to the 'energy gap', thus confirming that, as in the case of organic glasses [9, 10], these techniques make it possible to reach the lower-lying configurations of a-Si.

In view of the present results, we consider that: a) the usual claim [20,21] that amorphous tetrahedral semiconductors obtained by ion implantation constitute the model material to be compared with theoretical models is largely unfounded and b) the qualitative conclusions reached for the particular case of a-Si can be extrapolated to other tetrahedral semiconductors.

\section{Acknowledgements}

This work was partially funded by the Spanish MICINN (MAT2006-11144 project and SB2006-0062 post-doctoral contract) and by the Generalitat de Catalunya (Contract No. 2009SGR-185, 2009-SGR-770).

\section{References}

[1] P. G. Debenedetti et al., Nature 410, 259 (2001).

[2] S. Roorda et al., Phys. Rev. B 44, 3702 (1991).

[3] F. Kail et al., Appl. Phys. Lett. 97, 031918 (2010).

[4] D. Beeman et al., Phys. Rev. B 32, 874 (1985).

[5] T. Saito et al., J. Non-cryst. Solids 50, 271 (1982).

[6] E. P. Donovan et al., Appl. Phys. Lett. 55, 1516 (1989).

[7] S. M. Lee et al., Philos. Mag. B 60, 547 (1989).

[8] P. Roura et al., J. Appl. Phys. 104, 073521 (2008).

[9] S. F. Swallen et al., Science 315, 353 (2007).

[10] K. J. Dawson et al., Proc. Nat. Ac. Sci. 106, 15165 (2009).

[11] M. J. Cliffe et al., Phys. Rev. Lett. 104, 125501 (2010).

[12] N. Bernstein et al., Phys. Rev. B 74, 205202 (2006).

[13] J. M. Gibson et al., Phys. Rev. Lett. 105, 125504 (2010).

[14] P. Roura et al., Phys. Rev. B 73, 085203 (2006).

[15] P. Roura et al., Acta Mater. 57, 2098 (2009) and refs. therein.

[16] P. M. Voyles et al., J. Appl. Phys. 90, 4437 (2001).

[17] H. Oukris and N. E. Israeloff, Nature Phys. 6, 136 (2010).

[18] P. Roura et al., Thermochim. Acta 161, 522 (2011).

[19] K. Winer, Phys. Rev. B 35, 2366 (1987).

[20] G. D. Azevedo et al., Phys. Rev. B 68, 115204 (2003).

[21] K. Laaziri et al., Phys. Rev. Lett. 82, 3460 (1999). 
Figure 1. Solid lines: $\Delta \mathrm{H}_{\text {cryst }}$ for all samples analyzed (values of amorphized Si from [6]). Broken lines: $\Delta \mathrm{H}_{\text {cryst }}$ after two and five years after deposition. Shaded area: predicted range for strain energy.

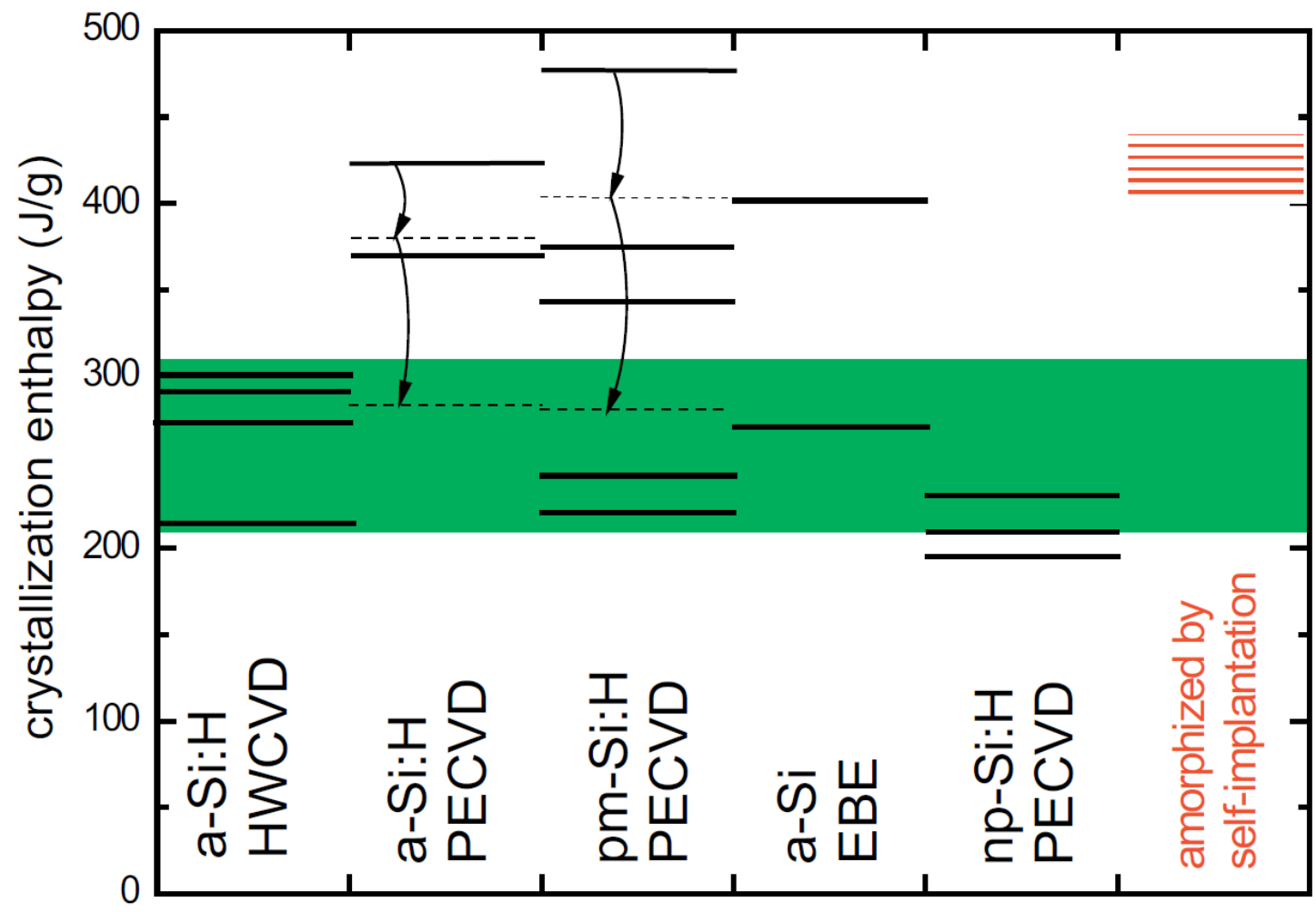


Figure 2. a) DSC curve of a HWCVD film; b) XRD curves for this film below and above the crystallization peak.

a)

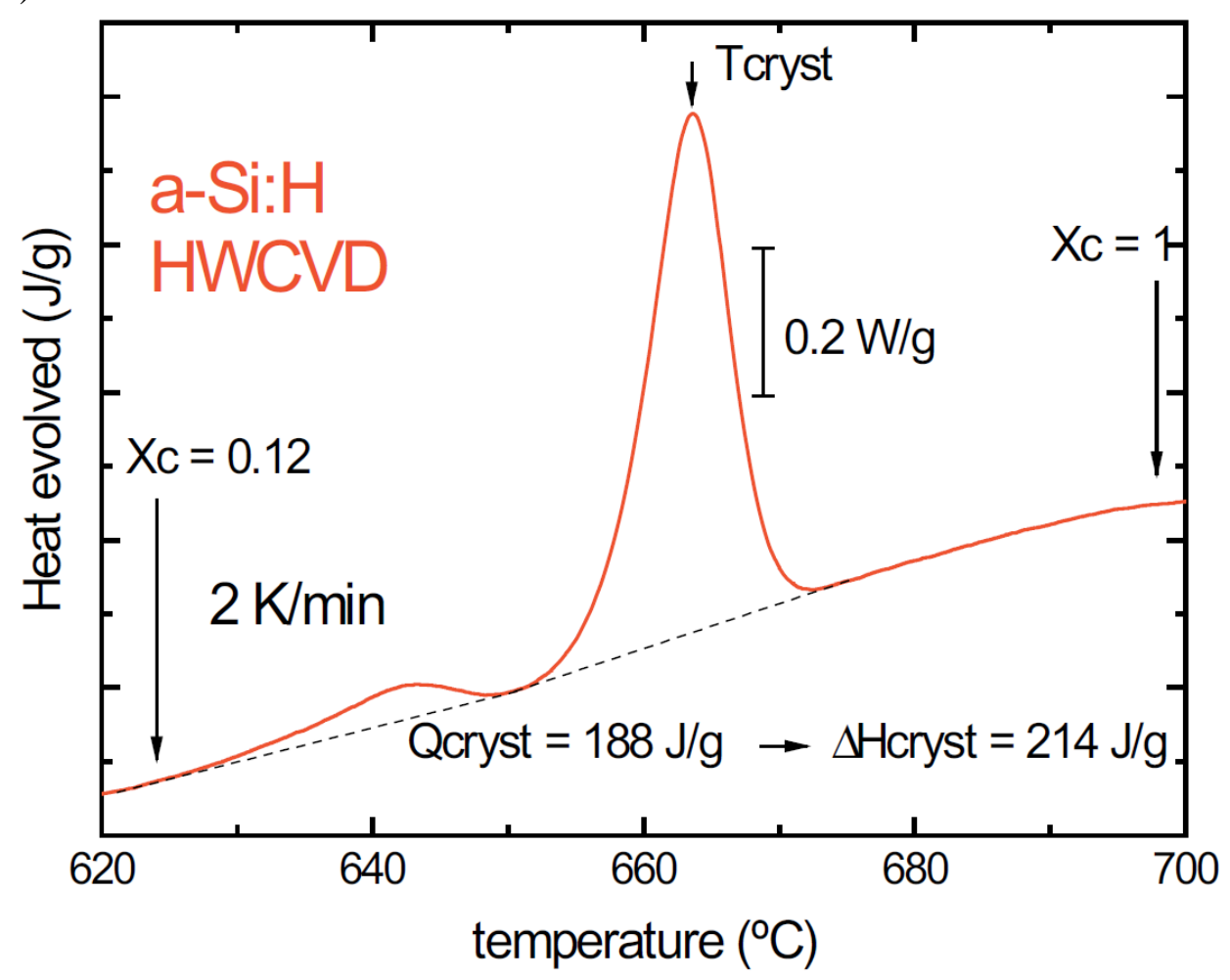

b)

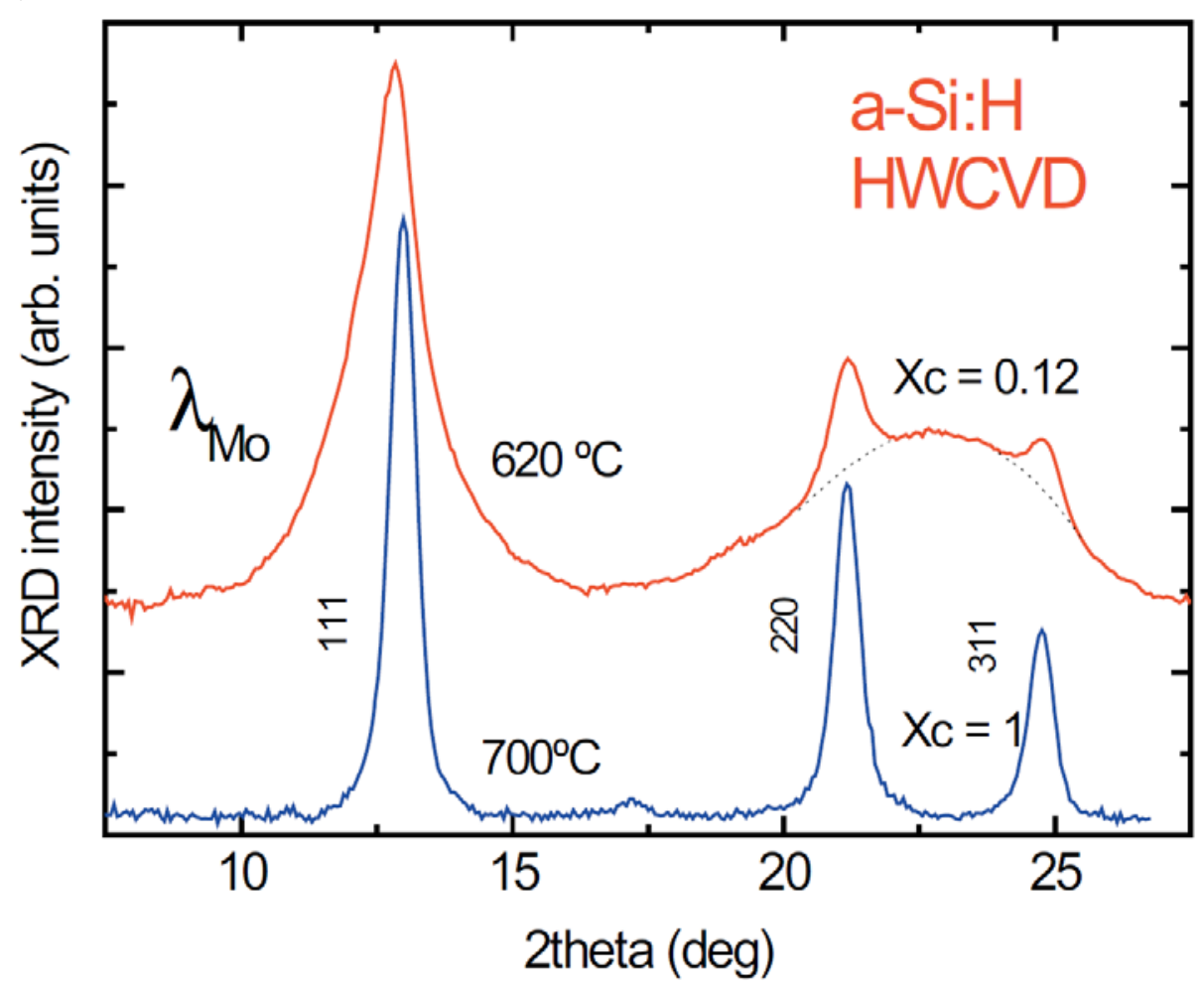

